\title{
Pharmacological Stimulation of Wnt/B-catenin Signaling Pathway Attenuates the Course of Thioacetamide-Induced Acute Liver Failure
}

\author{
Eva KOBLIHOVÁ ${ }^{1}$, Iveta MRÁZOVÁ ${ }^{2,3}$, Zdenka VAŇOURKOVÁ ${ }^{2}$, Hana MAXOVÁ ${ }^{3}$, Soňa \\ KIKERLOVÁ ${ }^{2}$, Zuzana HUSKOVÁ ${ }^{2}$, Miroslav RYSKA ${ }^{1}$, Jiří FRONĚK ${ }^{4}$, Zdenka \\ VERNEROVÁ ${ }^{2,5}$
}

${ }^{1}$ Department of Surgery, Second Faculty of Medicine, Charles University and Central Military Hospital, Prague, Czech Republic, ${ }^{2}$ Center for Experimental Medicine, Institute for Clinical and Experimental Medicine, Prague, Czech Republic, ${ }^{3}$ Department of Pathophysiology, Second Faculty of Medicine, Charles University, Prague, Czech Republic, ${ }^{4}$ Department of Transplantation Surgery, Institute for Clinical and Experimental Medicine, Prague, Czech Republic, ${ }^{5}$ Department of Pathology, Third Faculty of Medicine, Charles University, Prague, Czech Republic

Received October 30, 2018

Accepted October 29, 2019

Epub Ahead of Print December 19, 2019

\section{Summary}

Acute liver failure (ALF) is known for extremely high mortality rate, the result of widespread damage of hepatocytes. Orthotopic liver transplantation is the only effective therapy but its application is limited by the scarcity of donor organs. Given the importance in the liver biology of $\mathrm{Wnt} / \mathrm{\beta}$-catenin signaling pathway, we hypothesized that its stimulation could enhance hepatocyte regeneration and attenuate the course of thioacetamide (TAA)-induced ALF in Lewis rats. Chronic treatment with Wnt agonist was started either immediately after hepatotoxic insult ("early treatment") or when signs of ALF had developed ("late treatment"). Only $23 \%$ of untreated Lewis rats survived till the end of experiment. They showed marked increases in plasma alanine aminotransferase (ALT) activity and bilirubin and ammonia $\left(\mathrm{NH}_{3}\right)$ levels; plasma albumin decreased significantly. "Early" and "late" Wnt agonist treatment raised the final survival rate to $69 \%$ and $63 \%$, respectively, and normalized $\mathrm{ALT}, \mathrm{NH}_{3}$, bilirubin and albumin levels. In conclusion, the results show that treatment with Wnt agonist attenuates the course of TAA-induced ALF in Lewis rats, both with treatment initiated immediately after hepatotoxic insult and in the phase when ALF has already developed. Thus, the pharmacological stimulation of $W n t / \beta$-catenin signaling pathway can present a new approach to ALF treatment.

\section{Key words}

Acute liver failure - Thioacetamide • Wnt/ $\beta$-catenin signaling pathway

\section{Corresponding author}

Z. Vernerová, Department of Pathology, Third Faculty of Medicine, Charles University, Prague, Czech Republic. E-mail: vernerovazdena@seznam.cz

\section{Introduction}

The liver, one of the most biologically active organs with multiple functions, is indispensable for survival (Suchy 2009). Acute live failure (ALF) is a clinical syndrome resulting from a widespread damage of hepatocytes with resultant loss of liver function. While ALF is a rare organ disorder, mortality is extremely high (Bernal and Wendon 2013, Fyfe et al. 2018). The treatment of ALF remains supportive and presents a serious challenge, considering that only $45 \%$ of patients with ALF will recover. For the remaining $55 \%$ urgent orthotopic liver transplantation (OLT) is currently the only effective therapeutic approach (Fyfe et al. 2018, Patel et al. 2018). However, this treatment has limited application, primarily due to the scarcity of donor organs, especially the ones available on emergency notice. The 
other problem is the invasiveness of OLT procedure when applied in seriously ill ALF patients.

There is an obvious need to develop bridging techniques enabling survival of ALF patients until an organ is available and clinical situation improves sufficiently for OLT to be performed ("bridging to transplantation") or until liver function recovers ("bridging to recovery") (Fyfe et al. 2018, Patel et al. 2018, Zhang et al. 2018). In this context, it is emphasized that, unlike other visceral organs, the liver is capable of rapid regeneration: Normal cell turnover and regeneration processes after acute injury are mediated by proliferation of existing differentiated hepatocytes (Michalopoulos 2017). Therefore, the strategies to enable "bridging to transplantation" are focused on the stimulation of the extraordinary physiological regeneration capacity of the liver. A recognition is growing that $\beta$-catenin present in hepatocytes, a transcriptional coactivator that is controlled by Wnt ligand, plays an important role in almost every aspect of liver biology (Perugorria et al. 2019, Preziosi et al. 2018, Russell and Monga 2018). It was reported that stimulation of canonical Wnt-signaling that activates $\beta$-catenin is an important driver of liver regeneration following partial hepatectomy and ischemia/reperfusion (I/R) injury (Kuncewitch et al. 2013, Liu et al. 2015, Monga et al. 2001). Therefore, it seems plausible to assume that the stimulation of Wnt/ $\beta$-catenin signaling pathway could enhance the process of hepatocyte regeneration and attenuate the course of ALF.

On the other hand, it has to be considered that because of the pleiotropic nature of the $\mathrm{Wnt} / \beta$-catenin signaling pathway, its aberrant activation occurs also in hepatic pathologies and constant stimulation of the pathway by gene targeting methods could have undesirable effects (Ghosh et al. 2019, Perugorria et al. 2019, Russell and Monga 2018). Therefore, there is an obvious need for focused experimental studies that would examine the value and safety of novel therapeutic approaches for ALF.

For the induction of ALF and exploration of liver pathophysiology and new treatment approaches, the usage of hepatotoxic drugs is recommended, preferably of thioacetamide (TAA) (Butterworth et al. 2009). We further developed, optimized and characterized the TAA application in Lewis rats (Koblihova et al. 2014) and found it optimal for the purpose. The disadvantage of other hepatotoxic drugs, such as carbon tetrachloride and acetaminophen, is that after the lethal doses the animals die within 12 to $24 \mathrm{~h}$ (narrow therapeutic window) (Koblihova et al. 2014, Mehendale 2005).

It has been established in embryological studies that 2-amino-4-[3,4-(methlylenedioxy)benzylamino]-6(3methoxyphenly)pyrimidine, a small-molecule pyrimidine derivative, is an agonist of Wnt signaling pathway (Kaldis and Pagano 2009, Liu et al. 2005) which was efficiently activated after its intraperitoneal (i.p.) administration (Kuncewitch et al. 2013, Ma et al. 2016). Therefore, we used here this Wnt agonist for time-limited stimulation of the pathway; this approach should minimize potential detrimental off-target effects of alternative permanent stimulation of the Wnt/ $\beta$-catenin signaling pathway.

Lewis rats were chosen for the TAA induction of ALF because the model fulfills the crucial criteria needed: The liver damage is potentially reversible and reproducible, mortality is a direct consequence of liver damage, and the time between induction of the insult and death (therapeutic window) is sufficiently long (3-5 days) to apply some treatment measure.

With such an appropriate research model available, we have undertaken, first, to examine if chronic treatment with Wnt agonist started immediately after hepatotoxic insult would attenuate the course of TAA-induced ALF in Lewis rats. Second, to make the study more relevant to the clinical situation, we also examined whether the treatment would be effective in animals in the stage of developed widespread damage of hepatocytes. In attempt to further elucidate the role of Wnt/ $\beta$-catenin signaling pathway in the pathophysiology of TAA-induced ALF, we determined the expression of liver protein $\beta$-catenin in untreated animals with TAA-induced ALF and in animals exposed to chronic treatment with Wnt agonist, in both treatments protocols.

\section{Methods}

\section{Ethical approval, animals and chemicals}

The studies were performed in accordance with guidelines and practices established by the Animal Care and Use Committee of the Institute for Clinical and Experimental Medicine, Prague, which accord with the European Convention on Animal Protection and Guidelines on Research Animal Use. All the animals used in the study were housed in facilities accredited by the Czech Association of Laboratory Animal Care. The experiments were performed in male Lewis rats that were purchased from Charles River Laboratories (Velaz, 
Prague, Czech Republic) at the age of 9 weeks. Before starting experiments the rats were acclimatized in our vivarium for three weeks. The animals were kept on a 12-hour/12-hour light/dark cycle. Throughout the experiment, rats were fed a normal salt, normal protein diet $(0.45 \% \mathrm{NaCl}, 19-21 \%$ protein, SEMED, Prague, Czech Republic) and had free access to tap water. In order to maintain consistency and reproducibility of the results, Lewis rats were chosen, an inbred strain previously shown to be suitable for hepatocyte transplantation than can be performed without the need for post-transplantation immunosuppression (Koblihová et al. 2015); such treatment can alter the function of transplanted cells (Kawahara et al. 2010, Loukoupoulos et al. 2014). For comparison, we found that Wistar rats are more susceptible to the development of TAA-induced ALF (Koblihova et al. 2014) and exhibit a very narrow therapeutic window.

TAA (Sigma, Prague, Czech Republic) was dissolved in physiological saline and the appropriate dose was injected i.p. TAA has been known as a hepatotoxicant for more than 70 years, because it was first studied in 1948 in response to its detection in orange juice following its use as a fungicide in orange groves (Fitzhugh and Nelson 1948). TAA is biotransformed to thioacetamide sulfoxide which occurs along the cytochrome P-450 (CYP)-dependent pathway (CYP2E enzyme is mainly involved in this process).

In the body TAA is converted to thioacetamide disulfoxide, a toxic reactive metabolite that binds to liver macromolecules and dramatically increases the production of reactive oxygen species, leading to acute centrilobular liver necrosis (Koen et al. 2013). In the present study, freshly prepared TAA was administrated i.p. in two injections, on day 0 at 8:00 AM and 20:00 PM in the total amount $525 \mathrm{mg} \cdot \mathrm{kg}^{-1}$ of body weight (BW). This dose was chosen based on our studies evaluating the optimal doses of TAA for induction of ALF; we showed that after this dose all Lewis rats developed ALF and without treatment succumbed within first $48 \mathrm{~h}$ (Koblihova et al. 2014). Control rats received i.p. injections of physiological saline.

Wnt agonist (CID 11210285 hydrochloride, Sigma, Prague, Czech Republic) was administered i.p. at the dose of $5 \mathrm{mg} \cdot \mathrm{kg}^{-1}$ of BW dissolved in $20 \%$ dimethyl sulfoxide (DMSO) in normal saline once a day. Untreated animals received i.p. vehicle (20\% DMSO in saline) in the same amount as Wnt-treated animals (i.e. $0.5 \mathrm{ml}$ ). This dose of Wnt agonist was previously shown to effectively stimulate $\mathrm{Wnt} / \beta$-catenin signaling pathway in the liver (Kuncewitch et al. 2013, Ma et al. 2016).

\section{Experimental design}

Series 1: Effects of treatment with Wht agonist starting immediately after TAA administration ("early treatment protocol") on the course of TAA-induced ALF

Twenty-four hours before i.p. administration of TAA (“-24 h") blood sample (about $600 \mu \mathrm{l}$ ) was taken from the tail vein, for biochemical analyzes (Fuji DriveChem 4000 Analyser). Plasma levels of albumin, bilirubin, alanine aminotransferase (ALT) and aspartate aminotransferase (AST) activities, and ammonia level $\left(\mathrm{NH}_{3}\right)$ were determined. Blood samples for the same analyses were also taken 24, 48, 72, 96 and $168 \mathrm{~h}$ after the first administration of TAA. The follow-up period in this series was $168 \mathrm{~h}$ and at the end of experiments surviving animals were killed by an overdose of pentobarbital. Since during ALF development the animals' food and water intake decreased dramatically, $5 \%$ glucose solution, $2 \mathrm{ml} / 100 \mathrm{~g}$ of $\mathrm{BW}$, was administered subcutaneously every morning to prevent dehydration; our recent study demonstrated that this procedure is an effective remedy (Koblihová et al. 2014). The treatment with Wnt agonist was initiated $12 \mathrm{~h}$ after administration of the second dose of TAA and until the end of the experiment it was administered daily at 8:00 AM. The survival rate was monitored every $8 \mathrm{~h}$, BW was monitored every $24 \mathrm{~h}$ and blood samples were taken as described above.

The following experimental groups were examined:

1) Lewis rats + TAA + vehicle (Untreated Lewis rats with ALF) (initial $n=30$ )

2) Lewis rats + TAA + Wnt agonist (Lewis rats with ALF + Wnt agonist - "early treatment") (initial $\mathrm{n}=31$ )

3) Lewis rats + physiological saline + vehicle (Healthy Lewis rats) (initial $n=9$ )

4) Lewis rats + physiological saline + Wnt agonist (Healthy Lewis rats + Wnt agonist) (initial $n=10$ ).

Series 2: Effects of Wnt agonist on the course of TAA-induced ALF, with the treatment initiated in animals with fully developed ALF ("late treatment protocol")

The protocol as described for series 1 was used, except that the treatment with Wnt agonist was initiated $36 \mathrm{~h}$ after administration of the second dose of TAA, in the phase when signs of ALF are clearly visible, and 
within the next $24 \mathrm{~h}$ the animals begin to die (Koblihova et al. 2014, Koblihova et al. 2015). Thus, in this series the experimental group can be described as: Lewis rats + TAA + Wnt agonist (Lewis rats with ALF + Wnt agonist
- "late treatment") (initial $n=32$ ). The groups 1, 3 and 4 of series 1 served as controls.

The experimental design used in series 1 and 2 is outlined in Figure 1.
(A)

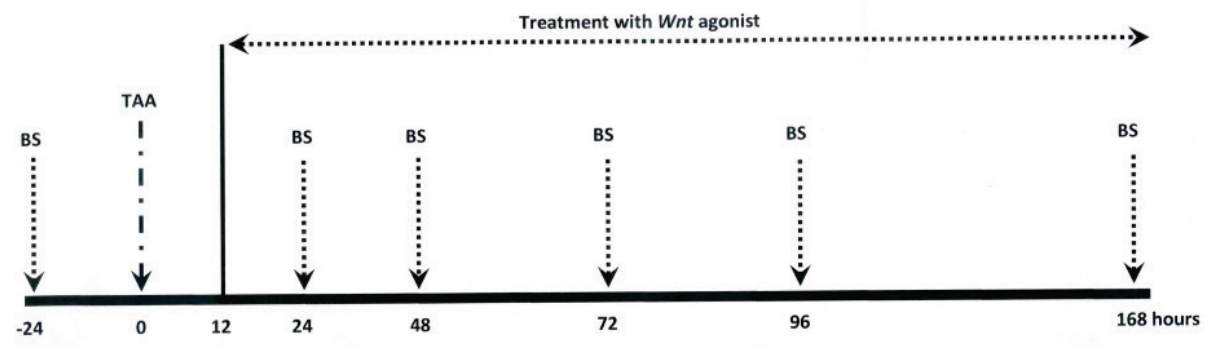

(B)
Fig. 1. An outline of experimental protocols: (A) early treatment protocol, (B) late treatment protocol. BS - blood sampling, TAA - administration of thioacetamide in two intraperitoneal injections in the total amount $525 \mathrm{mg} \cdot \mathrm{kg}^{-1}$ of body weight. Wnt agonist was administered once a day by intraperitoneal injection at the dose of $5 \mathrm{mg}^{-\mathrm{kg}^{-1}}$ of body weight.



Series 3: Effects of Wnt agonist on the liver expression of protein $\beta$-catenin, histological and immunohistochemical evaluation in animals exposed to "early treatment protocol"

The protocol was identical as described for series 1 , except that all surviving animals were killed $72 \mathrm{~h}$ after the first administration of TAA. In this series the following experimental groups were examined:

1) Untreated Lewis rats with $\operatorname{ALF}(n=7$ at $72 \mathrm{~h}$ after first TAA administration)

2) Lewis rats with ALF + Wnt agonist - "early treatment" $(\mathrm{n}=8$ at $72 \mathrm{~h}$ after first TAA administration)

3) Healthy Lewis rats ( $n=7$ at $72 \mathrm{~h}$ after first TAA administration)

4) Healthy Lewis rats + Wnt agonist ( $n=8$ at $72 \mathrm{~h}$ after first TAA administration)

Western blot analysis for quantification of liver $\beta$-catenin expression, histology and immunohistochemistry

The liver protein expression of $\beta$-Catenin, Phospho- $\beta$-catenin (Ser675), Non-phospho $\beta$-Catenin and Phospho- $\beta$-catenin (Ser33/37/Thr41) was determined as described in detail in previous studies (Bhushan et al. 2014, Apte et al. 2009). Briefly, liver tissue was homogenized 1:3 wt:vol in ice-cold RIPA lysis buffer containing $50 \mathrm{mM}$ Tris- $\mathrm{HCl} \mathrm{pH} 7.4,150 \mathrm{mM} \mathrm{NaCl}, 1 \%$ NP-40, $0.25 \%$ deoxycholic acid, $1 \mathrm{mM}$ EDTA; supplemented with protease inhibitor cocktail (SigmaAldrich, St. Louis, MO, USA), kept on ice for $30 \mathrm{~min}$ and centrifuged twice at $10000 \mathrm{x}$ g for $10 \mathrm{~min}$ at $4{ }^{\circ} \mathrm{C}$. Protein concentration in the supernatant was measured using Pierce BCA protein assay (Thermo Scientific, Waltham, MA, USA). In total, $50 \mu \mathrm{g}$ of protein was separated by sodium dodecyl sulfate polyacrylamide gel electrophoresis (SDS-PAGE) and transferred onto the polyvinyl difluoride (PVDF) membrane in transfer buffer at $100 \mathrm{~V}$ for $1.5 \mathrm{~h}$. Membranes were blocked with $5 \%$ BSA in TRIS buffered saline with Tween 20 (TBS-T) overnight at $4{ }^{\circ} \mathrm{C}$. After washing with TBS-T, the membranes were incubated with primary antibodies overnight at $4{ }^{\circ} \mathrm{C}$. The antibody dilutions were as follows: $\beta$-catenin, 1:500, Cell Signaling (Leiden, Netherlands);

Phospho- $\beta$-catenin (Ser675), 1:500, Cell Signaling (Leiden, Netherlands);

Non-phospho $\quad \beta$-catenin (Ser33/37/Thr41), 
1:1000, Cell Signaling (Leiden, Netherlands); Phospho- $\beta$-catenin (Ser33/37/Thr41), 1:250, Cell Signaling (Leiden, Netherlands).

After overnight incubation, the membranes were washed again and incubated with horseradish peroxidaseconjugated secondary antibody for $1 \mathrm{~h}$ at room temperature. After last washing, the immunoblots were exposed to SuperSignal West Dura Substrate (Thermo Scientific, Rockford, IL, USA) for chemiluminiscent detection. Relative densitometry was determined using ImageJ software (NIH, Bethesda, MD, USA). All protein data was normalized to the housekeeping protein $\beta$-actin. Phospho- $\beta$-catenin (Ser33/37/Thr41) is formed by glycogen synthase kinase 3 (GSK-3) and is responsible for the formation of $\beta$-catenin degradation complex which inhibits $\beta$-catenin activity ( $\mathrm{Wu}$ and $\mathrm{He} 2006$ ). Therefore, we analyzed the expression of non-phospho- $\beta$ catenin (Ser33/37/Thr41) (active) and phospho- $\beta$-catenin (Ser33/37/Thr41) (inactive) and their ratio.

Phospho- $\beta$-catenin (Ser675) is the result of protein kinase A (PKA) activity, which finally leads to Ser675 $\beta$-catenin accumulation in the nucleus and increases its transcriptional activity (Spirli et al. 2013), and it has been shown that its increased activity was associated with many detrimental actions (Ghosh et al. 2019, Perugorria et al. 2019).

Separate liver samples were washed with physiological saline and transferred into $4 \%$ formaldehyde. The sections stained with hematoxylineosin, peridodic acid Schiff reaction (PAS) and Masson trichrome stain were examined in a blind fashion and assessed using a semi-quantitative score as originally defined by Ishak et al. (1995) and employed in our previous study (Koblihová et al. 2014).

Immunohistochemical analysis was performed on five-micrometer-thick paraffin sections. Deparaffinization, rehydration, and antigen retrieval were performed by $\mathrm{CC} 1$ (prediluted; $\mathrm{pH} 8.5$ ) antigen retrieval solution (Roche), on the VENTANA BenchMark ULTRA automated slide stainer for $52 \mathrm{~min}$ at $98^{\circ} \mathrm{C}$. Specimens were incubated with primary monoclonal mouse antihuman Beta-catenin monoclonal antibody (clone $\beta$-catenin-1; Dako) using a concentration of 1:200 for $40 \mathrm{~min}$ at $37^{\circ} \mathrm{C}$, followed by visualization with the UltraView DAB IHC Detection Kit (Roche) for $12 \mathrm{~min}$. The specimens were then counterstained with haematoxylin and bluing reagent (Ventana) and coverslipped. Optimal staining conditions of a given antibody were determined using appropriate positive and negative controls. Positive results were based on linear membrane positivity of hepatocytes. The staining intensity was scored as follows: 0 (no stain), 1+ (weak), $2+$ (moderate) or $3+$ (strong membranous staining). The methods and evaluation procedures are in accordance with the technique employed previously by Apte $e t$ al. (2009).

\section{Statistical analysis}

Statistical analysis of the data was performed using Graph-Pad Prism software (Graph Pad Software, San Diego, CA, USA). Comparison of survival curves was performed by log-rank (Mantel-Cox) test followed by Gehan-Breslow-Wilcoxon test. ANOVA for repeated measurements, followed by Student-Newman-Keuls test, was performed for analysis of changes within the groups. Statistical comparison of other results was made by oneway ANOVA. Unless indicated otherwise, values are expressed as mean \pm S.E.M. A $\mathrm{P}<0.05$ was considered statistically significant.

\section{Results}

TAA administration in untreated Lewis rats dramatically decreased survival rate. The animals started to die $48 \mathrm{~h}$ after TAA administration; after $72 \mathrm{~h}$ only $43 \%$ and at the end of the experiment only $23 \%$ survived (Fig. 2). The treatment with Wnt agonist, both within the early and late treatment protocol, markedly improved the survival rate, to $86 \%$ and $87 \%$, respectively, when measured $72 \mathrm{~h}$ after TAA administration, and the respective final survival rates were $69 \%$ and $63 \%$. Remarkably, there was no significant difference in the course of survival rate between the rats treated with Wnt agonist within the early and late treatment protocols (Fig. 2A and 2B). All healthy untreated as well as healthy Lewis rats treated with Wnt agonist survived until the end of experiment (data not shown).

A marked increase in plasma $\mathrm{NH}_{3}$ was seen already in the first $24 \mathrm{~h}$ after TAA administration; this was significantly attenuated by early treatment with Wnt agonist (Fig. 3A). The increases in plasma $\mathrm{NH}_{3}$ levels in untreated animals were further augmented at 48 and at $72 \mathrm{~h}$ after TAA administration and the treatment with Wnt agonist either in the early or late treatment protocol substantially attenuated these increases.

Likewise, TAA administration caused a significant increase in plasma ALT activities and the treatment 
with Wnt agonist, both within the early and late treatment protocol, markedly attenuated these increases (Fig. 3B). As soon as $96 \mathrm{~h}$ after the first TAA administration, plasma ALT activity returned to levels observed under basal conditions and were not significantly different from the values observed in healthy animals. Plasma AST activity showed a similar pattern of changes (data not shown).

\section{A}

\section{Effects of early treatment}

- Untreated Lewis rats with ALF $\square$ Lewis rats with ALF + Wnt agonist

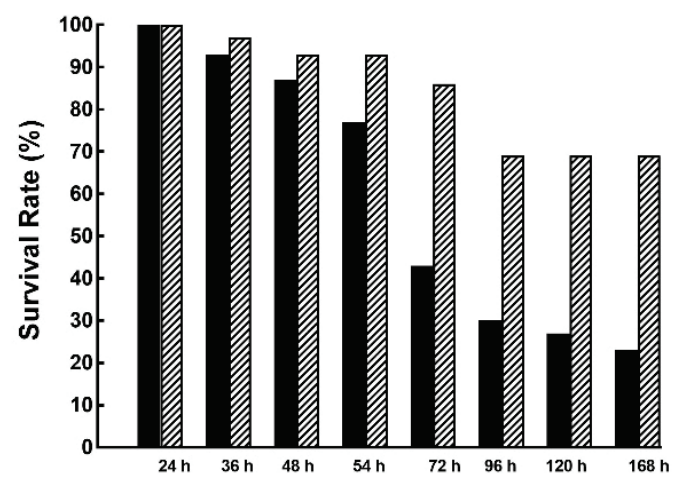

B

Effects of late treatment

Untreated Lewis rats with ALF . Lewis rats with ALF + Wnt agonist

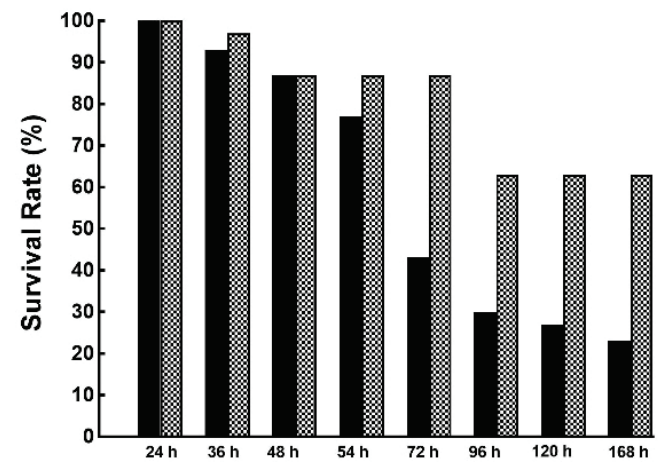

Fig. 2. Effects of early (A) and late (B) treatment with Wnt agonist on the survival rate in Lewis rats with acute liver failure (ALF) induced by thioacetamide administration. The survival rate of untreated Lewis rats with ALF was in both protocols significantly lower than in rats treated with Wnt agonist (analyzed by log-rank Mantel-Cox test followed by Gehan-Breslow-Wilcoxon test).

TAA administration elicited a significant elevation in plasma bilirubin levels and the treatment with Wnt agonist, both within the early and late treatment protocol, attenuated this increase, similarly as in the case of ALT activity (Fig. 4A).

Figure 4B shows that TAA treatment resulted first in a progressive decrease in plasma albumin levels,

followed by a recovery beginning at $72 \mathrm{~h}$ after administration. The treatment with Wnt agonist prevented the decreases in plasma albumin levels, both within the early and late treatment protocol.

There were no significant changes in any biochemical parameters in healthy Lewis rats treated with Wnt agonist throughout the experiment and therefore the data are not shown.

A
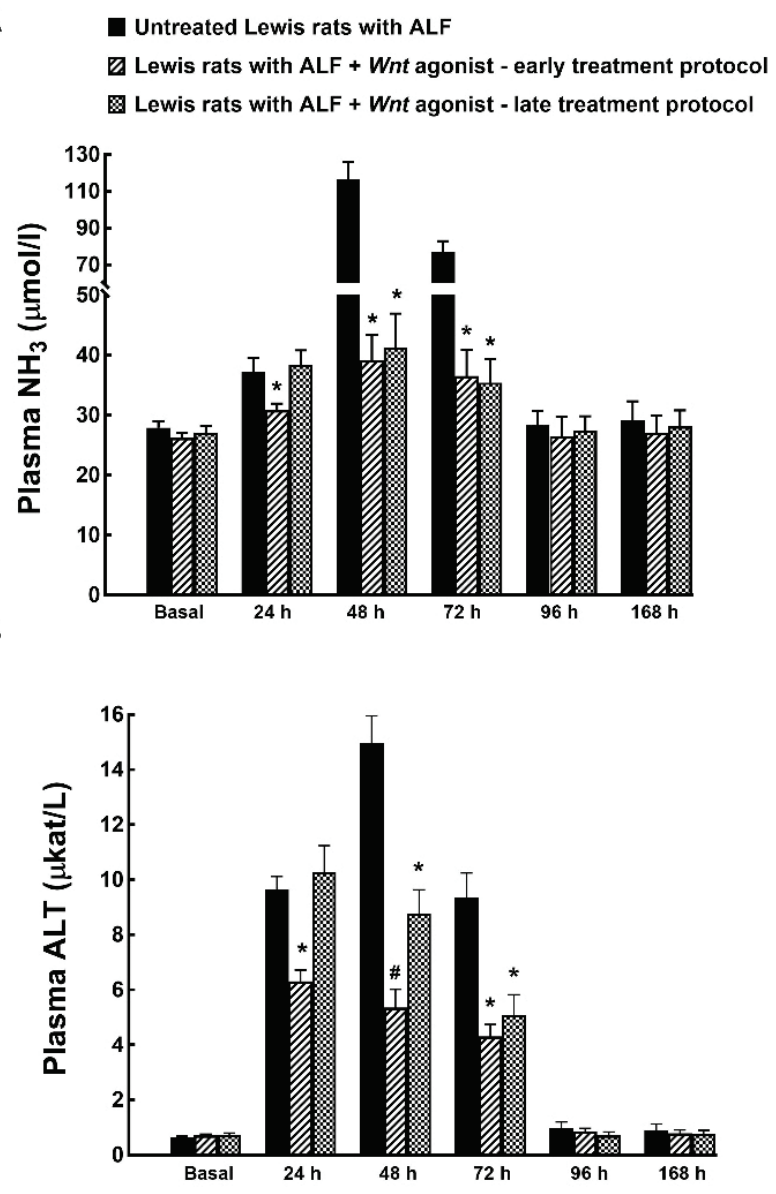

Fig. 3. Changes in plasma ammonia $\left(\mathrm{NH}_{3}\right)$ levels $(\mathbf{A})$ and plasma alanine aminotransferase (ALT) activities (B) in untreated Lewis rats with acute liver failure (ALF) induced by thioacetamide administration and in ALF rats exposed to early or late treatment protocol. ${ }^{*} \mathrm{P}<0.05$ versus the value for untreated ALF rats at the same time point. ${ }^{\#} \mathrm{P}<0.05$ versus all the other values at the same time point.

TAA administration did not cause any significant increase in the liver protein expression of total $\beta$-catenin, even though such a trend did occur. The treatment with Wnt agonist did not alter the total $\beta$-catenin liver protein expression in healthy animals or in the animals with TAA-induced ALF (Fig. 5A). There were no significant differences in liver protein expression of phospho- $\beta$-catenin (Ser675) in healthy animals or in 
animals with TAA-induced ALF, and the treatment with Wnt agonist did not change them (Fig. 5B).

TAA administration did not alter the liver protein expression of non-phospho- $\beta$-catenin (Ser33/37/ Thr41) and the treatment with Wnt agonist did not modify it in healthy animals or in those with TAA-induced ALF (Fig. 6A). In contrast, TAA administration resulted in marked decreases in the liver protein expression of phospho- $\beta$-catenin (Ser33/37/Thr41) and the treatment with Wnt agonist did not modify it in healthy animals or in those with TAA-induced ALF (Figure 6B). Figure 6C shows the balance between nonphospho- $\beta$-catenin (Ser33/37/Thr41) (active) and phospho- $\beta$-catenin (Ser33/37/Thr41) (inactive) levels of this form expressed as their ratio, and the data presented here show that animals with untreated TAA-induced ALF exhibit about 4-fold increase in this ratio. The treatment with Wnt agonist did not modify it, similarly in healthy animals and in animals with TAA-induced ALF.

\section{A}

Untreated Lewis rats with ALF Lewis rats with ALF + Wnt agonist - early treatment protocol Lewis rats with $A L F+W n t$ agonist - late treatment protocol

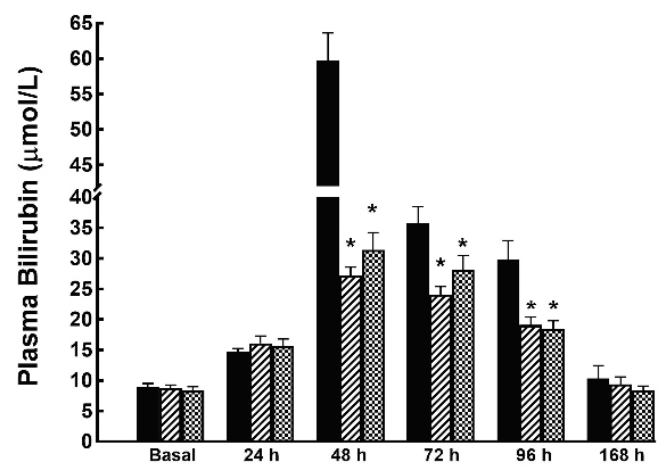

B

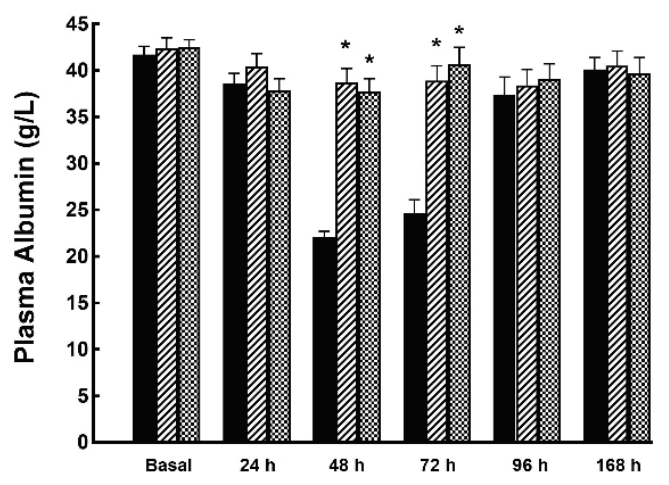

Fig. 4. Changes in plasma bilirubin (A) and albumin (B) levels in untreated Lewis rats with acute liver failure (ALF) induced by thioacetamide administration and in ALF rats exposed to early or late treatment protocol. ${ }^{*} \mathrm{P}<0.05$ versus the value for untreated ALF rats at the same time point.
Figure 7A shows that healthy Lewis rats had normal liver parenchyma and histological scoring revealed no damage (score 0 in all cases), and the treatment with Wnt agonist did not alter the morphology of liver parenchyma in healthy Lewis rats (Fig. 7B). TAA administration resulted in formation of numerous foci of centrilobular hemorrhagic necrosis with sporadic formation of bridging necrosis. At the end of our experiment, regenerative changes in the liver parenchyma dominated, particularly the resorption of centrolobular necroses and the bridging necroses mostly caused by the collapse of reticulin framework. Only sporadically were the traces of initial collagenization observed (Fig. 7C). In contrast, in animals with TAA-induced ALF treated with Wnt agonist the extent of liver damage was attenuated as compared with untreated animals with TAA-induced ALF (histological score $3.44 \pm 0.47$ vs. $5.14 \pm 0.36, \mathrm{p}<0.05$ ) (Fig. 7D).

A

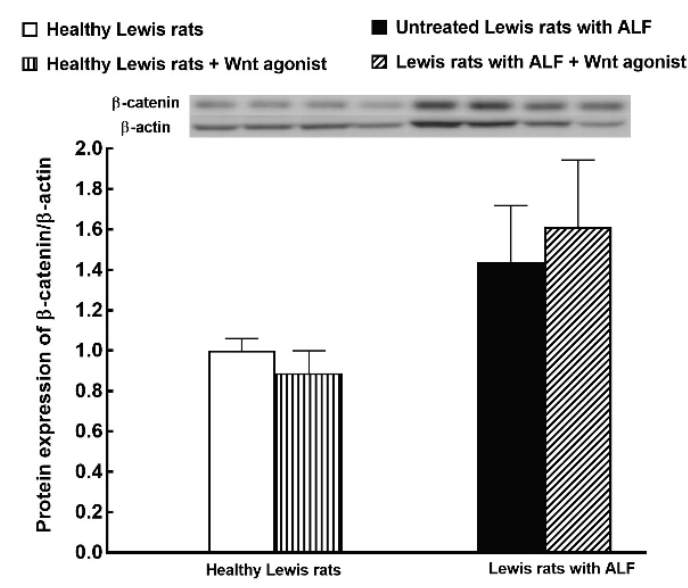

B
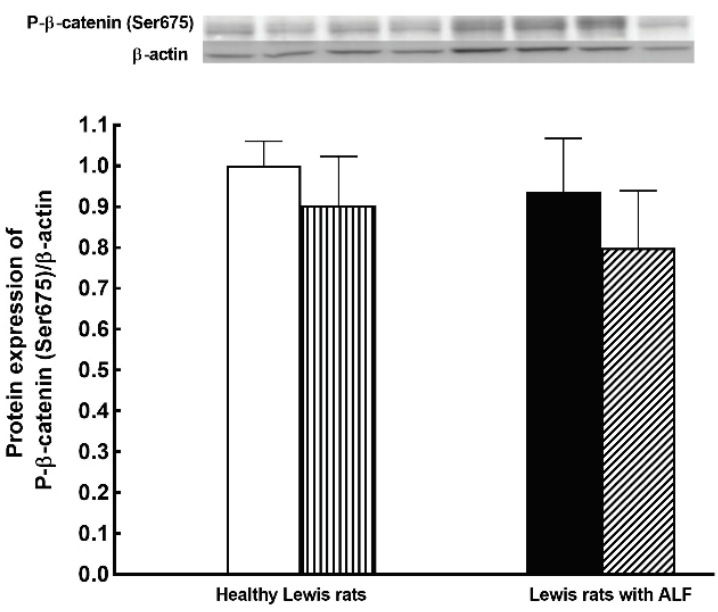

Fig. 5. Liver protein expression of total $\beta$-catenin (A) and phospho- $\beta$-catenin (Ser675) (B) in untreated healthy Lewis rats and untreated Lewis rats with acute liver failure (ALF) induced by thioacetamide administration, and in same group of animals treated with Wnt agonist. 
There were no significant differences in $\beta$-catenin staining intensity among the individual experimental groups and the representative images are shown in Figure 8.

A

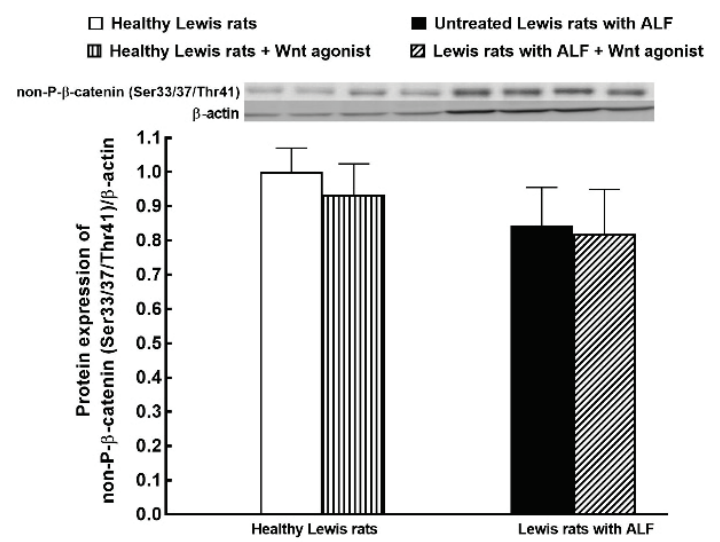

B

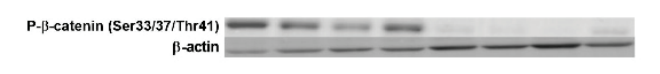

C
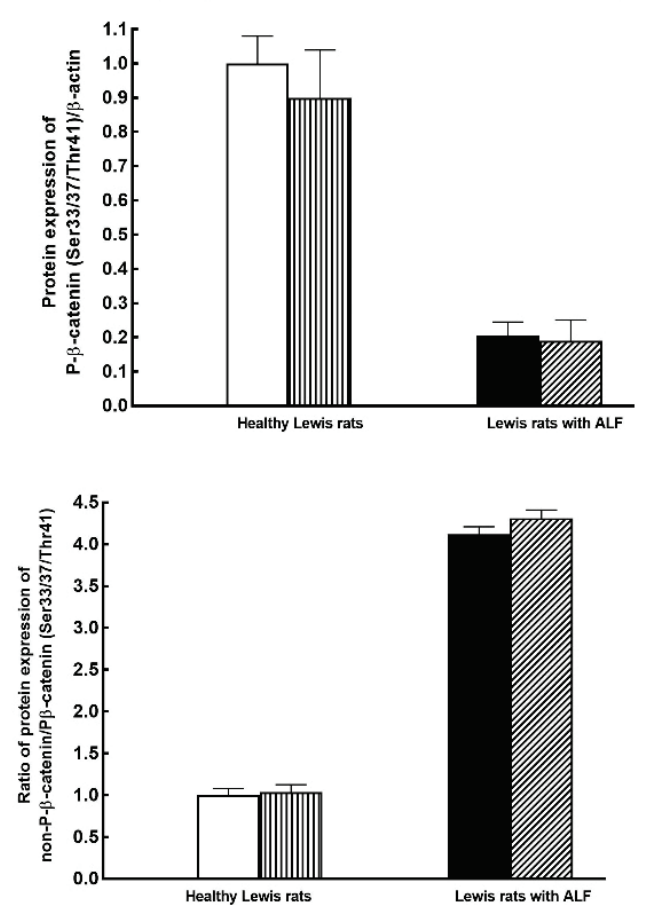

Fig. 6. Liver protein expression of non-phospho- $\beta$-catenin (Ser33/37/Thr41) (A), phospho- $\beta$-catenin (Ser33/37/Thr41) (B) and their ratio $(\mathbf{C})$ in untreated healthy Lewis rats and untreated Lewis rats with acute liver failure (ALF) induced by thioacetamide administration, and in the same group of animals treated with Wnt agonist. ${ }^{*} \mathrm{P}<0.05$ versus the value for healthy Lewis rats.

\section{Discussion}

The first important finding of the present study is that treatment with Wnt agonist started immediately after hepatotoxic insult attenuated the course of TAA-induced ALF in Lewis rats. This was clearly seen from improved survival rate and reduced degree of liver injury as indicated by lowering of plasma ALT, AST, $\mathrm{NH}_{3}$ and bilirubin levels, and also by lower histological score defining the degree of liver damage. The treatment also restored the biosynthetic function of the liver, because plasma albumin levels in surviving animals were not significantly different from those in healthy Lewis rats. Even more important is our second finding that all these beneficial effects of Wnt agonist were present also when treatment was initiated after signs of ALF became apparent. This finding enhances the clinical value of the results.

Taken together, these findings confirm our hypothesis that the pharmacological stimulation of the $\mathrm{Wnt} / \beta$-catenin signaling pathway can attenuate the course of TAA-induced ALF.

Hepatocytes are the major cell type of human liver, accounting for $80 \%$ of the parenchymal volume and are dominantly responsible for the organ's main functions: synthesis and metabolism of life-essential factors, detoxification, biotrasformation and excretion of foreign chemicals (Suchy 2009). Growing body of evidence indicates that the $\mathrm{Wnt} / \beta$-catenin signaling pathway in hepatocytes plays important role in various aspects of liver physiology (Russell and Monga 2018). A study of $\beta$-catenin knockout mice has demonstrated that the $\mathrm{Wnt} / \beta$-catenin signaling pathway plays a critical role in the embryonic (Haegel et al. 1995) but also in the postnatal liver development: on one hand, the loss of $\beta$-catenin in hepatocytes resulted in a significant decrease in liver growth (Tan et al. 2006), on the other hand, hepatocyte-specific overexpression of $\beta$-catenin caused a marked increase in liver size due to increased hepatocyte proliferation (Tan et al. 2005). Further studies have shown that the $\mathrm{Wnt} / \beta$-catenin signaling pathway importantly participates in all basic hepatocyte functions (Preziosi et al. 2018, Russell and Monga 2018). Moreover, the $\mathrm{Wnt} / \beta$-catenin signaling pathway is important for liver regeneration, as reported from studies in the two-thirds partial hepatectomy model (Monga et al. 2001). Within days, the remaining liver lobes enlarged due mostly to cell hyperplasia with some contribution of hypertrophy (Michalopoulos 2017). The treatment with Wnt agonist also accelerates regeneration in a partial liver transplant model (Ma et al. 2016). In addition, an important role of the $\mathrm{Wnt} / \beta$-catenin signaling pathway has been implicated in I/R injury, because knockout mice for hepatocyte $\beta$-catenin showed greater susceptibility to I/R liver injury whereas the mice with hepatocyte-specific 

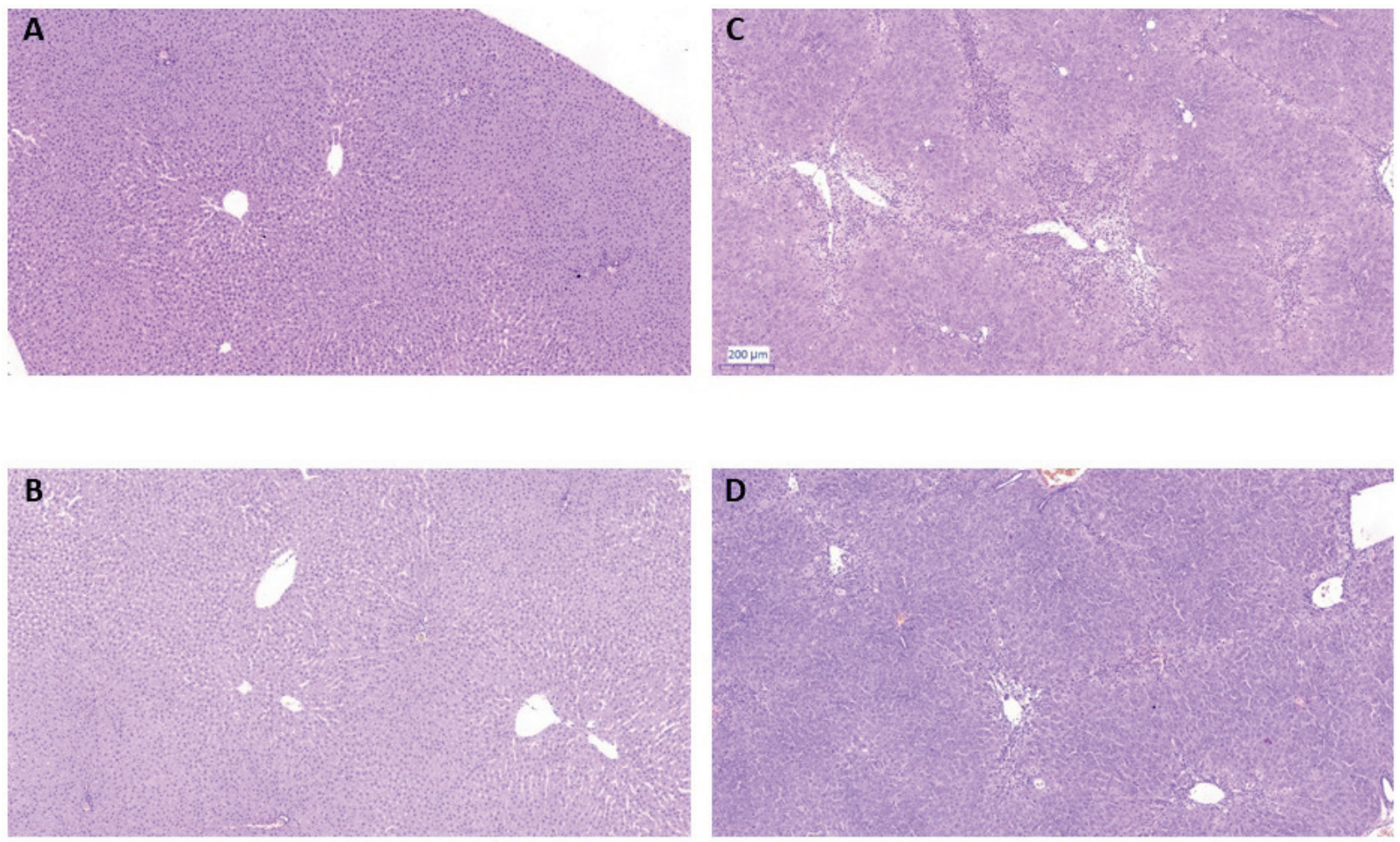

Fig. 7. Representative images of liver parenchyma (stained with hematoxylin-eosin, magnification $50 x$ ) in untreated healthy Lewis rats (A), healthy Lewis rats treated with Wnt agonist (B), untreated Lewis rats with acute liver failure (ALF) induced by thioacetamide (TAA) administration (C), and Lewis rats with TAA-induced ALF treated with Wnt agonist (D). Scale bar in the figure is $200 \mu \mathrm{m}$.
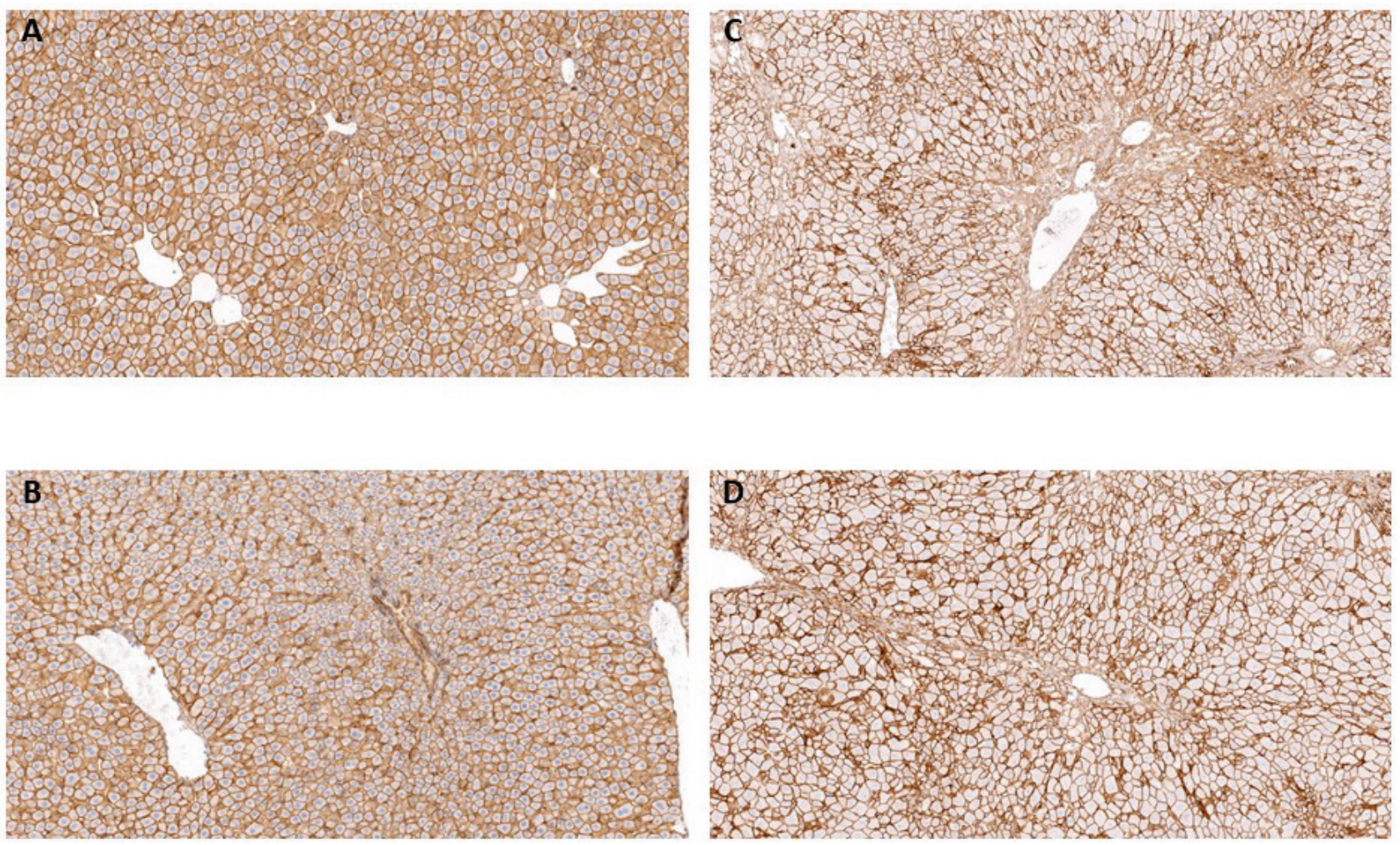

Fig. 8. Representative immunohistochemical imagines of $\beta$-catenin staining in the liver parenchyma (magnification 100x) in untreated healthy Lewis rats $(\mathbf{A})$, healthy Lewis rats treated with Wnt agonist $(\mathbf{B})$, untreated Lewis rats with acute liver failure (ALF) induced by thioacetamide (TAA) administration (C), and Lewis rats with TAA-induced ALF treated with Wnt agonist (D). 
Wnt/ $\beta$-catenin overexpression exhibited increased resistance to such injury (Lehwald et al. 2011). Furthermore, it has been documented that stimulation of $\mathrm{Wnt} / \beta$-catenin signaling pathway using the same agonist at the same dose as in our present study attenuated the $I / R$ injury and in a lethal model of hepatic $I / R$ injury the mortality rate was significantly reduced (Kuncewitch et al. 2013). Collectively, these findings prompted us to hypothesize that the pharmacological stimulation of the Wnt/ $\beta$-catenin signaling pathway may be a suitable approach to the treatment of ALF. This notion is further strengthened by findings that in non-lethal chemical ALF model $\beta$-catenin activation is an early event and it is vital for regeneration after APAP-induced ALF (Apte et al. 2009, Bhushan et al. 2014). However, administration of lethal doses of APAP, that are known to inhibit liver regeneration (Fyfe et al. 2018, Shan et al. 2018), was associated with suppression of liver $\beta$-catenin (Bhushan et al. 2014). Furthermore, it was demonstrated that Wnt/ $\beta$-catenin signaling pathway drives TAA-mediated heteroprotection against APAP-induced lethal ALF (Dadhania et al. 2017). Heteroprotection is a term to describe a situation when a minimal non-lethal dose of one hepatoxicant, in this case TAA, is administered $24 \mathrm{~h}$ before administration of a lethal dose of another hepatoxicant, in this case APAP. It was shown that the primary dose of non-lethal toxicant induces compensatory tissue repair, which protects against the lethal dose of the second toxicant (Mehenadale 1995); this resembles "mithridatism" i.e. the practice of protecting oneself against a poison by gradual selfadministration of non-lethal amounts (Mayor 2014). In this context, of special interest are our findings showing that even though the whole-liver $\beta$-catenin protein is not increased in untreated Lewis rats with TAA-induced ALF as compared with healthy Lewis rats, the balance between non-phospho- $\beta$-catenin (Ser33/37/Thr41) (active) and phospho- $\beta$-catenin (Ser33/37/Thr41) (inactive) is markedly increased, indicating that the formation of $\beta$-catenin degradation complex is substantially reduced and therefore the $\beta$-catenin activity in Lewis rats with TAA-induced ALF is enhanced compared with healthy animals. These findings are in agreement with the previous studies suggesting that early activation of $\beta$-catenin is critically important for liver regeneration after APAP-induced ALF (Apte et al. 2009, Bhushan et al. 2014). Based on this knowledge and our present Western blot, histological and immunohistochemical findings, early activation of $\beta$-catenin, particularly in pericentral hepatocytes of zone III, appears critically important for liver regeneration after hepatotoxic insult. We suggest that early activation of the Wnt/ $\beta$-catenin signaling pathway is a common sign of liver regeneration in APAP-induced as well as in TAA-induced ALF models.

This is of special interest, because hepatocyte transplantation (Tx) has recently emerged as a new possible approach to ALF treatment, either as "bridging to transplantation" or even "bridging to recovery" (Anderson and Zarinpar 2018, Iasante et al. 2018, Lee et al. 2018, Patel et al. 2018, Zhang et al. 2018). Initially, hepatocyte Tx has been successfully employed for the treatment of inborn metabolic liver diseases dependent on deficiency of a single hepatic enzyme or protein (e.g. Crigler-Najjar syndrome type I, urea cycle defects, and Wilson's disease) and can be corrected with engraftment of hepatocytes expressing the gene involved (Anderson and Zarinpar 2018, Iasante et al. 2018, Lee et al. 2018, Zhang et al. 2018). However, the first attempts of hepatocyte Tx in ALF were not always successful and it has been suggested that the number of viable hepatocytes currently transplanted might be sufficient to correct a single inborn defect, but not to provide full restoration of liver function (Anderson and Zarinpar 2018, Iasante et al. 2018, Lee et al. 2018, Zhang et al. 2018). In this context, it has been proposed that the significant limitation of hepatocyte $\mathrm{Tx}$ as a new approach for the treatment of ALF is their reduced viability (Anderson and Zarinpar 2018, Iasante et al. 2018, Lee et al. 2018, Zhang et al. 2018). Given the aforementioned importance of the Wnt/ $\beta$-catenin signaling pathway in liver biology and in view of our current findings, it can be reasoned that activation of this pathway could increase viability of the transplanted hepatocytes and improve the results of ALF treatment based on hepatocyte Tx. However, it has to be admitted that even though the treatment with Wnt agonist exhibited protective effects on the course of TAA-induced ALF, it was not reflected by the increased liver expression of non-phospho- $\beta$-catenin (Ser33/37/ Thr41) or increased balance between non-phospho- $\beta$ catenin (Ser33/37/Thr41) (active) and phospho- $\beta$-catenin (Ser33/37/Thr41) (inactive), nor did it alter $\beta$-catenin in the liver parenchyma. Taken together, our present findings indicate that the beneficial actions of the treatment with Wnt agonist are not simply the result of increased $\beta$-catenin expression in the liver parenchyma. Certainly, one could hypothesize that even though the 
treatment with Wnt agonist did not alter liver protein expression, it could increase the activity of the $\mathrm{Wnt} / \beta$-catenin signaling pathway. Evidently, comprehensive studies are needed to evaluate the precise mechanism(s) underlying the beneficial effects of the Wnt agonist treatment on the course of TAA-induced ALF, and our present results provide only the necessary background.

Aside from the above discussed drawbacks, our present study has one more important limitation. It was seen that untreated Lewis rats with TAA-induced ALF displayed important deterioration in the detoxification ability, as documented by marked increases in $\mathrm{NH}_{3}$ plasma levels, and the treatment with Wnt agonist dramatically improved the liver detoxification function. Again, the underlying mechanism(s) remain unknown. It is known that the toxic reactive metabolite of TAA impairs the function of the enzymes operative in the urea metabolic cycle (Mehandale 2005, Koen et al. 2013). Therefore one could assume that improvement of the liver detoxification function in the animals with TAA-induced ALF treated with Wnt agonist could be either the result of induction of glutamine synthetase activity or simply due to rapid restoration of the liver parenchyma and regeneration of zone 1 , which is the main location for the series of reactions known as the urea cycle in the liver (Suchy 2009). Again, instead of unsolicited speculations, future focused studies are needed to address this issue.

Nevertheless, it should be remembered that aberrant activation of the $\mathrm{Wnt} / \beta$-catenin signaling pathway has been implicated in the pathobiology of hepatocellular carcinoma (Jemal et al. 2010, Perugorria et al. 2019), hepatoblastoma (Purcell et al. 2011, Perugorria et al. 2019), and activation of hepatic stellate cells which have a role in liver fibrosis (Russell and Monga 2018) and, in general, accumulating evidence indicates that abnormalities in the $\mathrm{Wnt} / \beta$-catenin signaling pathway play an important role in the development of various cancers (Ghosh et al. 2019). Since the Wnt/ $\beta$-catenin signaling pathway can be involved both in liver regeneration as well as in its damage (Russell and Monga 2018, Perugorria et al. 2019), therapeutic strategies targeting this pathway must be carefully considered in order to avoid or at least minimize detrimental outcomes. In this context, of special interest is our finding that the treatment with Wnt agonist did not cause any detrimental effects in healthy animals. Nor did it induce any increase in liver phospho- $\beta$-catenin (Ser675), whose abnormal activity has been shown to be associated with tumorigenesis (Ghosh et al. 2019, Perugorria et al. 2019). Therefore, even though our follow-up period was relatively short, it seems that the treatment with Wnt agonist is safe, which agrees well with earlier reports (Kuncewitch et al. 2013, Liu et al. 2015, Ma et al. 2016). Thus, transient pharmacological activation of the $\mathrm{Wnt} / \beta$-catenin signaling pathway could be a novel approach for the enhancement of viability of transplanted hepatocytes and, consequently, for the improvement of the results of hepatocyte $\mathrm{Tx}$ in the treatment of ALF, both as "bridging to transplantation" and "bridging to recovery". Further studies are needed to fully evaluate this proposal and the present work provides a necessary background.

Our present results show that the treatment with Wnt agonist attenuates the course of TAA-induced ALF in Lewis rats, both when the treatment is initiated immediately after the hepatotoxic insult and when applied in the phase when the ALF has already developed. We believe that the pharmacological stimulation of the Wnt/ $\beta$-catenin signaling pathway should be considered in attempts to develop new therapeutic approaches or tools for the treatment of ALF.

\section{Conflict of Interest}

There is no conflict of interest.

\section{Acknowledgements}

This study was supported by Ministry of Health of the Czech Republic within the project for the development of research organization 00023001 (IKEM) and MO 2012 $(\mathrm{CMH})$ - institutional support.

\section{References}

ANDERSON TN, ZARINPAR A: Hepatocyte transplantation: past efforts, current technology, and future expansion of therapeutic potential. J Surg Res 226: 48-55, 2018. https://doi.org/10.1016/j.jss.2018.01.031

APTE U, SINGH S, ZENG G, CIEPLY B, VIRJI MA, WU T, MONGA SPS: Beta-catein activation promotes liver regeneration after acetaminophen-induced injury. Am J Pathol 175: 1056-1065, 2009. https://doi.org/10.2353/ajpath.2009.080976 
BERNAL W, WENDON J: Acute liver failure. $\mathrm{N}$ Engl Med 369: 2525-2534, 2013. https://doi.org/10.1056/NEJMra1208937

BHUSHAN B, WALESKY C, MANLEY M, GALLAGHER T, BORUDE P, EDWARDS G, MONGA SPS, APTE U: Pro-regenerative signaling after acetaminophen-induced acute liver injury in mice identified using a novel incremental dose model. Am J Pathol 184: 3013-3025, 2014. https://doi.org/10.1016/j.ajpath.2014.07.019

BUTTERWORTH RF, NORENBERG MD, FELIPO V, FERENCI P, ALBRECTH J, BLEI AT: Experimental models of hepatic encephalopathy: ISHEN guidelines. Liver Int 29: 783-788, 2009. https://doi.org/10.1111/j.1478$\underline{3231.2009 .02034 . \mathrm{x}}$

DADHANIA VP, BHUSHAN B, APTE U, MEHENDALE HM: Wnt/ $\beta$-catenin signaling drives thioacetamidemediated heteroprotection against acetaminophen-induced lethal liver injury. Dose Response 15: 1-13, 2017. https://doi.org/10.1177/1559325817690287

HAEGEL H, LARUE L OHSUGI M, FEDOROV L, HERRENKNECHT K, KEMLER R: Lack of $\beta$-catenin affects mouse development at gastrulation. Development 121: 3529-3537, 1995.

FITZHUGH OG, NELSON AA: Liver tumors in rats fed thiourea or thiocetamide. Science 108: 626-628, 1948. https://doi.org/10.1126/science.108.2814.626

FYFE B, ZALDANA F, LIU C: The pathology of acute liver failure. Clin Liver Dis 22: 257-268, 2018. https://doi.org/10.1016/j.cld.2018.01.003

GHOSH N, HOSSAIN U, MANDAL A, SIL PC: The Wnt signaling pathway: a potential therapeutic target against cancer. Ann N Y Acad Sci 1443: 54-74, 2019. https://doi.org/10.1111/nyas.14027

IANSANTE V, MITRY RR, FILIPPI C, FITZPATRICK E, DHAWAN A: Human hepatocyte transplantation for liver disease: current status and future perspectives. Pediatr Res 83: 232-240, 2018. https://doi.org/10.1038/pr.2017.284

ISHAK K, BAPTISTA A, BIANCHI L, CALLEA F, DE GROOTE J, GUDAT F, DENK H, DESMET V, KORB G, MACSWEEN RNM, PHILLIPS MJ, PORTAMNN BG, POULSEN H, SCHEUER PJ, SCHMID M, THALER H: Histological grading and staging of chronic hepatitis. J Hepatol 22: 696-699, 1995. https://doi.org/10.1016/0168-8278(95)80226-6

JEMAL A, CENTER MM, DESANTIS C, WARD EM: Global patterns of cancer incidence and mortality rates and trends. Cancer Epidemiol Biomakr Prev 19: 1893-1907, 2010. https://doi.org/10.1158/1055-9965.EPI-10-0437

KALDIS P, PAGANO M: Wnt signaling in mitosis. Dev Cell 17: 749-750, 2009. https://doi.org/10.1016/j.devcel.2009.12.001

KAWAHARA T, TOSO C, DOUGLAS DN, NOURBAKHSH M, LEWIS JT, TYRRELL DL, LUND GA, CHURCHILL TA, KNETEMAN NM: Factors affecting hepatocyte isolation, engrafment, and replication in an in vivo model. Liver Transpl 16: 974-982, 2010. https://doi.org/10.1002/lt.22099

KOBLIHOVA E, MRÁZOVÁ I, VERNEROVÁ Z, RYSKA M: Acute liver failure induced by thioacetamide: selection of optimal dosage in Wistar and Lewis rats. Phys Res 63: 491-503, 2014.

KOBLIHOVA E, LUKŠAN O, MRÁZOVÁ I, RYSKA M, ČERVENKA L: Hepatocyte transplantation attenuates the course of acute liver failure induced by thioacetamide in Lewis rats. Phys Res 64: 689-700, 2015.

KOEN YM, SARMA D, HAJOVSKY H, GALEVA NA, WILIAMS TD, STAUDINGER JL, HANZLIK RP: Protein targets of thioacetamide metabolites in rat hepatocytes. Chem Res Toxicol 26: 564-574, 2013. https://doi.org/10.1021/tx400001x

KUNCEWITCH M, YANG WL, MOLMENTI E, NICASTRO J, COPPA GF, WANG P: Wnt agonist attenuates liver injury and improves survival after hepatic ischemia/reperfusion. Schock 39: 3-10, 2013. https://doi.org/10.1097/SHK.0b013e3182764fe8

LEE CA, SINHA S, FITZPATRICK E, DHAWAN A: Hepatocyte transplantation and advancements in alternative cell sources for liver-based regenerative medicine. J Mol Med 96: 469-481, 2018. https://doi.org/10.1007/s00109018-1638-5

LEHWALD N, TAO GZ, JANG KY SORKIN M, KNOEFEL WT, SYLVESTER KG: Wnt- $\beta$-catenin signaling protects against hepatic ischemia and reperfusion injury in mice. Gastroenterology 141: 707-718, 2011. https://doi.org/10.1053/j.gastro.2011.04.051 
LIU J, WU X, MITHCELL B, KINTNER C, DING S, SCHULTZ PG: A small-molecule agonist of the Wnt signaling pathway. Angew Chem Int Ed Engl 44: 1987-1990, 2005. https://doi.org/10.1002/anie.200462552

LIU B, ZHANG R, TAO G, LEHWALD NC, LIU B, KOH Y, SYLVESTER KG: Augmented Wnt signaling as a therapeutic tool to prevent ischemia/reperfusion injury in liver: preclinical studies in a mouse model. Liver Transpl 21: 1533-1542, 2015. https://doi.org/10.1002/lt.24331

LOUKOPOULOS I, SFINIADAKIS I, PILLAI A, KONSTANTOULAKIS M, ANDROULKAKIS G, BONATSOS V, ZOGRAFOS G, PAPLOIS A: Mycophenalote mofetil and sirolimus in hepatocyte transplantation in an experimental model of toxic acute liver failure. J Invest Surg 27: 205-213, 2014. https://doi.org/10.3109/08941939.2013.879967

MA Y, LV X, HE J, LIU T, WEN S, WANG L: Wnt agonist stimulates liver regeneration after small-for-size liver transplantation in rats. Hepatol Res 46: E154-E164, 2016. https://doi.org/10.1111/hepr.12553

MAYOR A: Mithridates of Pontus and his universal antidote. In: History of Toxicology and Environmental Health, Toxicology in Antiquity. WEXLER P (ed.), Elsevier, Amsterdam, 2014, pp 21-33. https://doi.org/10.1016/B978-0-12-800045-8.00004-6

MEHENDALE HM: Toxicodynamics of low level toxicant interactions of biological significance: inhibition of tissue repair. Toxicology 105: 251-266, 1995. https://doi.org/10.1016/0300-483X(95)03220-A

MEHENDALE HM: Tissue repair: an important determinant of final outcome of toxicant-induced injury. Toxicol Pathol 33: 41-51, 2005. https://doi.org/10.1080/01926230590881808

MICHALOPOULOS GK: Hepatostat: liver regeneration and normal liver tissue maintenance. Hepatology 65: 1384-1392, 2017. https://doi.org/10.1002/hep.28988

MONGA SP, PEDIADITAKIS P, MULE K, STOLZ DB, MICHALOPOULOS GK: Changes in Wnt/ $\beta$-catenin pathway during regulated growth in rat liver regeneration. Hepatology 33: 1098-1109, 2001. https://doi.org/10.1053/jhep.2001.23786

PATEL P, OKORNKWO N, PYRSOPOULOS NT: Future approaches and therapeutic modalities for acute liver failure. Clin Liver Dis 22: 419-427, 2018. https://doi.org/10.1016/j.cld.2018.01.011

PERUGORRIA MJ, OLAIZOLA P, LABIANO I, ESPARZA-BAQUER A, MARZIONI M, MARIN JJG, BUJANDA L, BANALES JM: Wnt- $\beta$-catenin signaling in liver development, health and disease. Nat Rev Gastroenterol Hepatol 16: 121-136, 2019. https://doi.org/10.1038/s41575-018-0075-9

PREZIOSI M, OKABE H, PODDAR M, SINGH S, MONGA SP: Endothelial Wnts regulate $\beta$-catenin signaling in murine liver zonation and regeneration: a sequel to the Wnt-Wnt situation. Hepatol Commun 2: 845-860, 2018. https://doi.org/10.1002/hep4.1196

PURCELL R, CHILDS M, MAIBACH R, MILES C, TURNER C, ZIMMERMANN A, SULLIVAN M: HGF/c-Met related activation of $\beta$-catenin in hepatoblastoma. J Exp Clin Cancer Res 30: 96, 2011. https://doi.org/10.1186/1756-9966-30-96

RUSSELL JO, MONGA SP: Wnt/ß-catenin signaling in liver development, homeostasis, and pathobiology. Annu Rev Pathol 13: 351-378, 2018. https://doi.org/10.1146/annurev-pathol-020117-044010

SHAN S, SHEN Z, SONG F: Autophagy and acetaminophen-induced hepatotoxicity. Arch Toxicol 92: 2153-2161, 2018. https://doi.org/10.1007/s00204-018-2237-5

SPIRLI C, LOCATELLI L, MORELL CM, FIOROTTO R, MORTON SD, CADAMURO M, FABRIS L, STRAZZABOSCO M: PKA dependent p-Ser-675- $\beta$-catenin, a novel signaling defect in a mouse model of congenital hepatic fibrosis. Hepatology 58: 1713-1723, 2013. https://doi.org/10.1002/hep.26554

SUCHY FJ: Hepatobiliary function. In: Medical Physiology. BORON WF, BOULPAEP EL (eds $2^{\text {nd }}$ edition), Saunders Elsevier, Philadelphia, PA, 2009, pp 980-1008. https://doi.org/10.1016/B978-1-4160-3115-4.50049-4

TAN X, APTE U, MICSENYI A, KOTSAGRELOS E, LUO JH, RANGANATHAN S, MONGA DK, BELL A, MIHCALOPOULOS GK, MONGA SP: Epidermal growth factor receptor: a novel target of the Wnt/ $\beta$-catenin pathway in liver. Gastroenterology 129: 285-302, 2005. https://doi.org/10.1053/j.gastro.2005.04.013

TAN X, BEHARI J, CIEPLY B, MICHALOPOULOS GK, MONGA SP: Conditional deletion of $\beta$-catenin reveals its role in liver growth and regeneration. Gastroenterology 131: 1561-1572, 2006. https://doi.org/10.1053/j.gastro.2006.08.042 
WU G, HE X: Threonin 41 in beta-catenin serves as a key phosphorylation relay residue in beta-catenin degradation. Biochemistry 45: 5319-5323, 2006. https://doi.org/10.1021/bi0601149

ZHANG J, ZHAO X, LIANG L, LI J, DEMIRCI U, WANG S: A decade of progress in liver regenerative medicine. Biomaterials 157: 161-176, 2018. https://doi.org/10.1016/j.biomaterials.2017.11.027 